\title{
GENERAL REVIEW OF COSMOLOGICAL THEORIES
}

\author{
F. HOYLE
}

Institute of Theoretical Astronomy, Cambridge, Great Britain

\begin{abstract}
Quantum effects in the early stages of some cosmological models are considered. Interactions between particles can be expressed in terms of the Dirac delta function, and the implications of the existence of both positive and negative frequencies in the Fourier expansion of this function are discussed.
\end{abstract}

The most general form for the space-time metric is

$$
\mathrm{d} s^{2}=c^{2} \mathrm{~d} t^{2}-Q^{2}(t)\left[\frac{\mathrm{d} r^{2}}{1-k r^{2}}+r^{2} \mathrm{~d} \Omega^{2}\right]
$$

when the Universe is taken to be homogeneous and isotropic, where $k$ can be $0, \pm 1, t$ is the time, $r$ a radial comoving coordinate and $\mathrm{d} \Omega$ is an element of solid angle. The case $k=0$ is Euclidean spatially but when different values of the time are considered all three-space distances are changed by the scale factor $Q(t)$. The $r$ coordinate of a particle (or galaxy) stays the same at all times but the product $r Q$ changes with time. The observer is usually taken to have $r=0$.

The case $k=0$ separates closed universes with finite volume, $k=+1$, from open universes, $k=-1$. It is the $k=0$ case that gives the value +0.5 for the deceleration parameter $q_{0}$. This case also relates the mean density $\varrho$ with $H$, defined by

$$
H=\dot{Q} / Q
$$

through

$$
\varrho=\begin{aligned}
& 3 H^{2} \\
& 8 \pi G
\end{aligned} .
$$

The present value of $c / H$ is $\sim 10^{28} \mathrm{~cm}$ and the corresponding density is $\sim 10^{-29} \mathrm{~g}$ $\mathrm{cm}^{-3}$. This density is usually described as that necessary 'to close the universe', by which we may understand the following:

The mass $m$ inside a sphere of radius $c / H$ is given by

$$
m=\frac{4 \pi}{3}\left(\frac{c}{H}\right)^{3} \varrho \simeq 10^{55} \mathrm{~g}
$$

when $\varrho \simeq 10^{-29} \mathrm{~g} \mathrm{~cm}^{-3}$ and $c / H \simeq 10^{28} \mathrm{~cm}$. Using (3) and (4) it is easily shown that

$$
\frac{2 G m}{c^{2}(c / H)}=1 \text {. }
$$

That is to say, using the density of the $k=0$ cosmology, the Schwarzschild radius of an isolated sphere of radius $c / H$ coincides with itself. This shows that general relativity becomes important in the $k=0$ cosmology as distances approach $c / H$. General rela- 
tivity is less important as distances approach $c / H$ in the $k=-1$ case because $\varrho$ is then less than (3). Conversely, general relativity is more important for such distances in the $k=+1$ case because $\varrho$ is greater than (3).

We might note that the angular deflection of electromagnetic radiation produced by a body of mass $m$ and radius $r$ is determined by the relativistic parameter $2 \mathrm{Gm} / \mathrm{c}^{2} r$. If the Universe were inhomogeneous on a scale $c / H$ by more than $\sim 0.1 \%$ in the $k=0$ case this would produce too great an anisotropy in the microwave background. A similar restriction applies for $k= \pm 1$, although the change of density from (3) in these cases alters the numerical value of the maximum permitted large scale inhomogeneity.

Table I gives related values of $t, Q, \varrho$ and of the temperature $T_{b}$ of the microwave background, assumed primaeval. The values are again for the $k=0$ case. Similar tables can be constructed for the $k= \pm 1$ cases.

TABLE I

\begin{tabular}{lcccl}
\hline$t(\mathrm{~s})$ & $Q$ & $\varrho\left(\mathrm{g} \mathrm{cm}^{-3}\right)$ & $T_{b}(K)$ & \multicolumn{1}{c}{$\begin{array}{l}\text { Communication } \\
\text { range }(\mathrm{Mpc})\end{array}$} \\
\hline $3.10^{17}$ & Unity (Standard) & $10^{-29}$ & 3 & 3000 \\
$3.10^{11}$ & $10^{-4}$ & $10^{-17}$ & $3.10^{4}$ & 30 \\
$3.10^{8}$ & $3.10^{-6}$ & $3.10^{-13}$ & $10^{6}$ & 1 \\
$\sim 10^{-103}$ & $\sim 10^{-61}$ & $\sim 10^{154}$ & $\sim 3.10^{61}$ &
\end{tabular}

I will come later to the significance of the right-hand column.

The last line of the table brings out the point that all models based on (1), and satisfying Einstein's equations without a cosmological constant, are singular in origin. Some fifteen years ago the question was raised by Heckmann and Schücking (1955, 1956) as to whether departure from isotropy through rotation could obviate such a singular origin. Narlikar (1963) used the following argument to answer this interesting question in the negative provided closed timelike lines were excluded. The field equations for such a Universe with uniform rotation were similar to those given by considerations of Newtonian cosmology. The problem could be analysed completely in the latter case, not only for rotation but also for shear and the existence of a singularity could be proved. A similar demonstration in terms of general relativity was made by Hawking and Ellis (1965) following methods used by Penrose (1965). This subject has recently been reviewed by Hawking and Penrose (1970). The conclusion is the one just stated: models with zero kosmical constant, satisfying general relativity, with homogeneity and with a suitable Cauchy surface possess a singularity.

The next question to consider is whether the existence of a singularity can properly be discussed within classical physics. All problems in physics must be discussed quantum mechanically whenever the action $S$ associated with the system in question is 
not large compared to $\hbar$. The action associated with the metric properties of spacetime is

$$
\frac{1}{16 \pi G} \int_{v} R \sqrt{-g} \mathrm{~d}^{4} x,
$$

where $R$ is the scalar curvature. Since $R=8 \pi G T / c^{4}$ where $T$ is the trace of the energymomentum tensor, we have $R=0$ for an empty universe. Since $T$ is also zero for transverse electromagnetic fields, such as the microwave background, the action is zero for a universe containing only transverse fields.

The presence of matter gives non-zero action, but this can be made small for the first brief moment of the universe. Suppose we consider the particular matter that at present we observe within distance $c / H \simeq 10^{28} \mathrm{~cm}$. From (4) the mass of this material $\sim 10^{55} \mathrm{~g}$ (using $k=0$ for definiteness) and the rest energy associated with this mass is $\sim 10^{76} \mathrm{erg}$. The action corresponding to $0 \leqslant t$ is $\sim 10^{76} t \mathrm{~g} \mathrm{~cm}^{2} \mathrm{~s}^{-1}$, which for $t \simeq 10^{-103} \mathrm{~s}$ is $\sim \hbar$. The situation at this value of $t$ is shown in the last line of the above table. To obtain the spatial scale of this mass distribution $\left(\sim 10^{55} \mathrm{~g}\right)$ we multiply the present scale, $\sim 10^{28} \mathrm{~cm}$, by the appropriate value of $Q$. This gives $\sim 10^{-33} \mathrm{~cm}$ which $\sim\left(G \hbar / c^{3}\right)^{1 / 2}$, and there are theoretical reasons why this should be so.

What do quantum considerations imply? That there was no unique space-time. We are free to consider space-time metrics, not satisfying Einstein's equations, provided (6), computed for such a metric, does not become large compared to $\hbar$.

Returning to models satisfying (1), all such models have communication horizons. Suppose a light signal was emitted at $t=0$ by the observer $(r=0)$. The last column of Table I gives the radial coordinate $r$ reached by the light at the corresponding values of $t$ (for the case $k=0$ ). Not until $t \simeq 3.10^{8} \mathrm{~s}$ was there communication through a cluster of galaxies, for example $(1 \mathrm{Mpc})$. Not until the present day does the communication range become as large as $3000 \mathrm{Mpc}$. Models satisfying (1) therefore require a remarkable degree of initial homogeneity. We receive the microwave background radiation from distances $\sim 10^{28} \mathrm{~cm}$, or at least we do if it is primaeval. The radiation we receive now comes from regions with which we had no previous communication. Since the radiation from opposite directions in the sky is the same to within $\sim 0.1 \%$ the initial condition must have been homogeneous to within $\sim 0.1 \%$.

Misner (1969) has sought to avoid the assumption of initial homogeneity by a drastic departure from (1). Perhaps initially the universe departed from (1) so violently that all the regions we now observe were in good communication with each other near $t=0$. I have the impression that the quantum considerations referred to above may be relevant at this point. Such considerations may help the situation because the lack of a unique metric might well establish channels of communication that would be impossible classically. The situation could be analogous to the passage of a particle through a potential barrier; this is possible in quantum theory because classicallyforbidden paths for the particle occur.

I turn now to cosmological problems concerned with the 'modern epoch' when the 
expansion factor $Q$ was not a great deal smaller than its present value. Apart possibly for the microwave background all our observational material refers to this modern epoch.

It is remarkable that the $\log N-\log S$ curve for radio sources requires that most sources appeared recently. If the radio luminosity function is everywhere the same then there were not many sources before $Q \sim \frac{1}{3}$. This has suggested to several workers that the Lemaitre closed model, based on (1) with $k=+1$, and including a suitably chosen cosmical constant in the field equations, should be investigated as a likely model for the universe. The $q_{0}$ value for this model is $<-1$, so the model would be ruled out if $q_{0}>0$. Personally, I believe one can question whether the galaxies used in the $q_{0}$ analysis are sufficiently alike intrinsically for this disproof to be taken as decisive.

The Lemaître model in question possesses an antipodal point. Light emitted by an object near the observer's antipode can reach the observer in two ways, either directly or by the null geodesic through the antipode. This is for the homogeneous situation. Petrosian and Salpeter (1968) noticed a peculiar effect due to inhomogeneities near the antipode. A single inhomogeneity can be regarded as displacing the antipode by a distance of the order of its own size. For a source at a distance from the antipode of the same order as the scale of the inhomogeneity the direction of the light track to the observer via the antipode is then greatly changed, although the direct route is scarcely affected. In a more complicated situation with many homogeneities there can be several non-direct routes to the observer. This produces the ghost images discussed by Petrosian and Salpeter. It is of interest that several QSS at almost the same red-shift, 1.955, are in fact known.

I turn now to an approach to cosmology which for me has great weight. A photon emitted by particle $a$ travels to particle $b$ and is absorbed only when the points $A$ and $B$ of emission and absorption are such that $s_{A B}^{2}=0$. This suggests an interaction $\delta\left(s_{A B}^{2}\right)$ in terms of the Dirac delta function. This function is time symmetric, however. In terms of a.Fourier expansion it contains both positive and negative frequencies,

$$
\delta\left(s^{2}\right)=\frac{1}{2 \pi} \int_{-\infty}^{\infty} \exp \left(-i \omega s^{2}\right) \mathrm{d} \omega .
$$

Such a symmetric form does not represent the required interaction correctly. To obtain the correct form we must omit the negative frequencies, $\omega<0$. It is this omission which leads to atoms jumping spontaneously downward, and not spontaneously upward. But why? Why not omit the positive frequencies, $\omega>0$ ? Then atoms would jump spontaneously upward. In effect, the electrodynamic arrow of time would point in the opposite sense to the expansion of the universe.

Let us try the symmetric form (7). Then from what has just been said, electrodynamic propagation occurs both backward and forwards. Our two particles $a$ and $b$ have interactions with other particles both forwards and backwards. We have the following situation: 
(i) Particles $a$ and $b$ interact with the past;

(ii) Particles $a$ and $b$ interact with the future;

(iii) Particles $a$ and $b$ interact between themselves through the function $\delta\left(s^{2}\right)$.

This may be compared with the usual situation in which

(i) Particles $a$ and $b$ interact with the past;

(ii) Particles $a$ and $b$ interact between themselves through the positive frequency part of $\delta\left(s^{2}\right), \delta_{+}\left(s^{2}\right)$.

These situations can be brought into consonance with each other provided it can be shown that the effect of (ii) in the first case is just to cancel the negative frequency part of $\delta\left(s^{2}\right)$, so that (ii) and (iii) in the first case become identical to (ii) in the second case.

This equivalence can be proved (Hoyle and Narlikar, 1969, 1970) provided the future light cone is completely opaque to radiation and provided the past light cone is not completely opaque. Friedman cosmologies fail to meet both these requirements - the future light cone is transparent in these cosmologies and the past light cone is opaque just the wrong way round. The direction of electrodynamic causality is reversed.

The basic requirement is that the proper density does not tend to zero in the future, and that in the past the density did not tend to infinity - there was no singularity. A finite density, not necessarily steady, possibly oscillating by a moderate factor, is necessary. The future light cone is then opaque because radiation into the future is red-shifted. An advanced wave propagating into the past would, on the other hand, be blue-shifted. It could penetrate a finite density to $t \rightarrow-\infty$ because of the blueshifting, provided the finite density was not too high.

If the density is oscillatory, the natural time of oscillation is $\sim H^{-1}$. All our astrophysical evidence relating to cosmology would refer to the current cycle. Evolutionary effects if they exist, for example in the space density of radio sources, would be related to the phase of the cycle. It might be supposed, for example, that the space density of radio sources would be greater when the average mass density was increasing than when it was falling.

If these considerations are correct the microwave radiation cannot be attributed to the origin of the universe. The microwaves must be astrophysical in their nature.

\section{References}

Hawking, S. W. and Ellis, G. F. R.: 1965, Phys. Letters 17, 246.

Hawking, S. W. and Penrose, R.: 1970, Proc. Roy. Soc. London 314 A, 529.

Heckmann, O. and Schücking, E.: 1955, Z. Astrophys. 38, 95.

Heckmann, O. and Schücking, E.: 1956, Z. Astrophys. 40, 81.

Hoyle, F. and Narlikar, J. V.: 1969, Ann. Phys. 54, 207.

Hoyle, F. and Narlikar, J. V.: 1970, Ann. Phys., in press.

Misner, C. W.: 1969, Phys. Rev. Letters 22, 1071.

Narlikar, J. V.: 1963, Monthly Notices Roy. Astron. Soc. 126, 203.

Penrose, R.: 1965, Phys. Rev. Letters 14, 57.

Petrosian, V. and Salpeter, E. E.: 1968, Astrophys. J. 151, 411. 
The above review of cosmological theories, confined to a presentation of 45 minutes, was of necessity an outline. For other issues not explicitly discussed above see the following:

Doroshkevich, A. G. and Novikov, I. D.: 1970, preprint.

Gunn, J. E.: 1967, Astrophys. J. 150, 737.

Hawking, S. W.: 1969, Monthly Notices Roy. Astron. Soc. 142, 129.

Jacobs, K. C.: 1968, Astrophys. J. 153, 661.

Jacobs, K. C.: 1969, Astrophys. J. 155, 379.

Kalatnikov, I. M. and Lifshitz, E.: 1970, Phys. Rev. Letters 24, 76.

Kontowski, R.: 1969, Astrophys. J. 155, 89.

Kristian, J. and Sachs, R.: 1966, Astrophys. J. 143, 379.

MacCallum, M. A. H. and Ellis, G. F. R.: 1970, Comm. Math. Phys.

Matese, J. J. and O'Connell, R. F.: 1970, Astrophys. J. 160, 451.

Matzner, R. A.: 1969, Astrophys. Space Sci. 4, 459.

Misner, C. W.: 1968, Astrophys. J. 151, 431.

Misner, C. W.: 1970, Phys. Rev. 186, 1319.

Misner, C. W.: 1970, Phys. Rev. 186, 1328.

Rees, M. J. and Sciama, D. W.: 1968, Nature 217, 511.

Sachs, R. and Wolfe, A. M.: 1967, Astrophys. J. 147, 73.

Saunders, P. T.: 1968, Monthly Notices Roy. Astron. Soc. 141, 427.

Saunders, P. T.: 1969, Monthly Notices Roy. Astron. Soc. 142, 213.

Stewart, J. M.: 1969, Monthly Notices Roy. Astron. Soc. 145, 347.

Thorne, K. S.: 1967, Astrophys. J. 148, 51.

Zeldovich, Y. B.: 1970, JETP Letters 11, 300.

\section{Discussion}

Heckmann: Two remarks, a mathematical and an historical: (1) I would like to state that there exist spaces with negative curvature, $k=-1$, which are closed; (2) The question whether, in Newtonian cosmology, rotation could remove the singularity was answered in the affirmative by Heckmann and Schücking in 1954/55. Narlikar's negative result of 1964 is based on specialized equations.

Narlikar: The point raised by Professor Heckmann has been discussed by Zeldovitch and myself in two notes in the Monthly Notices Roy. Astron. Soc. The difference arises from the solutions of the source-free potential equation $\nabla^{2} \varphi=0$.

Ne'eman: I suspect that your argument is based upon time-reversal symmetry. However, we now know for sure that $\mathrm{T}$ is violated in $K^{0}$ decay. Even though this is not due to electromagnetism, it will affect your propagator in higher-order corrections. However, it might still turn out that what is crucial in this reasoning is CPT rather than just T (microscopically), and CPT might still be conserved. 